\title{
tic\&société
}

Vol. 2, $n^{\circ} 1 \mid 2008$

Tic et santé

\section{Les blogs « extimes » : analyse sociologique de l'interactivité des blogs}

Sébastien Rouquette

\section{(2) OpenEdition \\ Journals}

Édition électronique

URL : http://journals.openedition.org/ticetsociete/412

DOI : 10.4000/ticetsociete.412

Éditeur

Association ARTIC

Référence électronique

Sébastien Rouquette, "Les blogs « extimes » : analyse sociologique de l'interactivité des blogs »,

tic\&société [En ligne], Vol. 2, n 1 | 2008, mis en ligne le 13 octobre 2008, consulté le 01 mai 2019. URL http://journals.openedition.org/ticetsociete/412 ; DOI : 10.4000/ticetsociete.412 


\title{
Les blogs « extimes » : analyse sociologique de l'interactivité des blogs
}

\author{
Sébastien ROUQUETTE \\ Maître de conférences en communication \\ Université Clermont-Ferrand II \\ Université Clermont-Ferrand II \\ IUP Information Communication \\ 34, Avenue Carnot \\ 63000 Clermont-Ferrand
}

Sébastien Rouquette est maître de conférences en communication de I'IUP Infocom, Université de Clermont-Ferrand II. II est membre des équipes de recherche "Communication et Solidarité " (Clermont-Ferrand II) et "Ceisme » (Paris III). Après des premiers travaux sur l'espace médiatique télévisé, il travaille actuellement sur Internet comme « média » différent (son caractère fragmenté, individualisé, son nouveau rapport au territoire, etc.). 


\title{
Les blogs « extimes » : analyse sociologique de l'interactivité des blogs
}

\begin{abstract}
Résumé :
Le premier objectif de cet article est de mieux comprendre les motivations initiales des blogueurs de blogs personnels. Le second est plus fondamentalement d'expliquer la perception globalement positive qu'ont les blogueurs des réactions des internautes. Finalement, l'engouement que cette nouvelle activité suscite fait ressortir quatre types de motivations : témoigner, dévoiler sa personnalité (éventuellement créative), donner son avis, écrire. Ainsi, le succès des blogs extimes tient alors en partie à leur capacité à prendre en charge des besoins d'expression variés dans la population. Mais il tient aussi au caractère jugé "valorisant », "enrichissant " des réactions reçues par les blogueurs à la lecture de leurs billets.
\end{abstract}

\section{Mots-clés}

blog personnel, interactivité, analyse de blog, sociologie des blogueurs, présentation de soi en ligne

\begin{abstract}
The primary objective of this article is to understand the initial motivations of bloggers of personal blogs. The second is to explain the globally positive perception that bloggers have about reactions of the Internet users. Finally, four types of motivations dominate: give a testimony, reveal his(her) personality, give her opinion, write. The success of those blogs can thus be explained by the positive reactions received by bloggers about their blogs.
\end{abstract}

\section{Keywords}

Personal Blogs, interactivity, website analysis, sociology of bloggers, on-line identity

\section{Resumen}

Este artículo tiene tres objetivos. El primero es entender mejor por qué la implicación de los internautas/lectores es determinante en el contenido del blog personal. El segundo, busca explicar la percepción globalmente positiva que los bloguers tienen de las reacciones de los internautas. Por último, la admiración que suscita esta nueva actividad permite considerar 
Les blogs « extimes » : analyse sociologique de l'interactivité des blogs

cuatro tipos de motivaciones: mostrar, descubrir su personalidad (eventualmente creativa), dar su opinión y escribir. Es por eso que el éxito de los blogs extimes se debe, en parte, a su capacidad de tener en cuenta las variadas necesidades de expresión de las personas. También es debido al caracter valorizante y enriquecedor de las respuestas que reciben los titulares del blog, a sus escritos.

\section{Palabras clave}

blog personal, interactividad, análisis del blog, sociología de los bloguers, presentación en línea 


\section{Introduction : l'internaute au centre du dispositif des blogs extimes}

En peu d'années, le développement des plates-formes de blogs gratuits a profondément modifié la pratique de publication d'un journal en ligne. Même si le nombre de blogs est difficile à évaluer aujourd'hui en raison du caractère dynamique, évolutif et décentralisé de la blogosphère, des moteurs de recherche spécialisés et de méta-annuaires comme technorati.com ou blogpulse.com comptabilisent entre 50 et 85 millions de blogs à partir de juin 2007 (Lin et Halavais, 2006).

Cette forte croissance explique (partiellement) le constat selon lequel il n'existe pas de définition stabilisée de «l'objet blog». Faut-il insister sur la publication antéchronologique des billets, sur le principe d'autopublication, sur les limites techniques de cet objet hypermédia, sur la grande ouverture des blogs vers le reste du réseau (par exemple: Pledel, 2006, pp. 252-255; Trédan, 2005, pp. 1-2 ; Miller et Shepherd, 2004) ? Malgré cette diversité, chacun s'accorde sur l'importance des « posts »; autrement dit sur le caractère central de l'interactivité des blogs.

La polysémie de ce terme est telle (Bourdeloie, 2000), l'évolution des procédés d'interaction si rapide, que l'interactivité ne peut se satisfaire d'une seule définition (Seguy, 1999). En fait, deux grandes significations ont été distinguées par de nombreux auteurs soucieux de lutter contre les effets de simplification et surtout de généralisation abusive que la confusion entre deux niveaux rend possible.

Dans un premier sens, il s'agit de l'interactivité technique de la relation homme-machine au sens où l'entendent les informaticiens, c'est-à-dire un "dialogue caractéristique de nouvelles générations d'outils capables d'échanger des informations symboliques avec leurs utilisateurs humains » (Thierry, 2002, p. 248). Celle-ci se « caractérise par un échange d'informations sous une forme symbolique évoluée (très souvent linguistique) obligatoirement effectuée entre un être humain et une machine capable de traiter des données » (Thierry, 2002, p. 249). Elle ne concerne donc pas les échanges humains (forums, chats, etc.)

Or, par un effet de glissements sémantiques successifs, l'interactivité de relation (informatique) a très rapidement été confondue avec l'interactivité de contenu, celle qui englobe les questions de coproduction des contenus et d'influence directe des remarques, des billets des internautes sur les articles en 
Les blogs « extimes » : analyse sociologique de l'interactivité des blogs

ligne. Cette seconde interactivité ressort d'une autre logique: celle d'une influence réciproque et non unidirectionnelle ${ }^{1}$.

Or, pour un webmaster ${ }^{2}$ comme pour un internaute, les implications de ces deux formes d'interactivité diffèrent radicalement. On ne peut réduire l'interactivité médiatique d'un magazine à celle de la diversité des rubriques accessibles, l'interactivité démocratique d'un site politique à celle de la variété des parcours de lecture possibles, sauf à confondre «les modalités techniques de l'interactivité entre humain et machines avec les modalités sociales de l'interaction entre individus » (Proulx et Sénécal, 1995, p. 239). Dans un blog plus particulièrement, seul le second niveau, le plus ambitieux, est révélateur du caractère " ouvert », « participatif », etc. du blog extime consulté.

Or, si plusieurs recherches empiriques ont porté sur le contenu ou les motivations de blogueurs de sites personnels (Lenhart et Fox, 2006, pp. 1-33; Nowson et Oberlander, 2006, pp. 627-634 ; Allard et Vandenderghe, 2003, pp. 190-219), une analyse de ces blogs sous l'angle de leur interactivité a pour intérêt de mieux comprendre la dynamique de cette écriture de soi et de cette présentation aux autres qu'implique cette interactivité directe, quasi-immédiate et permanente avec les internautes.

En cela, le blog est d'abord une pratique sociale plus qu'une activité personnelle (Nardi et Ali., 2004, pp. 222-231), ou plutôt une activité sociale à la fois interactive, à distance et écrite.

\footnotetext{
${ }^{1}$ Michel Sénécal compare les distinctions formulées entre ces deux formes d'interactivité dans plusieurs disciplines scientifiques, toutes se basant sur ce principe d'influence réciproque : "actions réciproques entre deux êtres, deux phénomènes (éducation) [...]"; "action et réaction, c'est l'influence réciproque exercée entre deux individus, entre un individu et un groupe ou entre les individus d'un même groupe (médecine)"; ou encore "réciprocité de l'influence des comportements entre deux ou plusieurs individus ou groupes [..] (psychologie) » (2006, p. 136).

2 "Plus les entreprises médiatiques font appel à des techniques prétendument interactives, plus il leur est facile de promouvoir l'idée que ces médias seront ainsi davantage « démocratiques » et du coup, plus ouverts, accessibles et transparents » (Souchier et Jeanneret, 2002).
} 


\section{1 - Objectifs et méthodologie d'analyse de l'interactivité des sites}

\subsection{La notion de blog extime}

La notion de blog extime ${ }^{3}$ a été préférée à celle de blog personnel pour deux raisons.

D'une part, elle insiste plus sur la démarche d'exposition publique de la vie privée, volontaire et consciemment intégrée dans les billets rédigés. La généralisation des blogrolls (liste de liens favoris en page d'accueil) et des fonctions " commentaires » ont - de fait - clôt l'un des débats ayant émergé aux débuts des «cybercarnets » et autres « journaux intimes » en ligne : celui des modifications des frontières entre vie publique et vie privée, de l'exposition de l'intime dans le cyberespace et les reformulations de l'intimité que ces expositions entraînent (Fosseux, 2001, pp. 15-30). Aujourd'hui, la question s'est déplacée tant de nombreux blogueurs reconnaissent eux-mêmes que cet exercice ne s'apparente en rien à celui d'une publication d'un journal intime. D'autant que, comme l'explique un spécialiste du genre autobiographique, "le diariste $^{1}$ [blogueur] ne suit que certains fils de son existence, ce qui fait problème, ou le passionne : le journal [en ligne] n'est nullement un récit complet de sa vie, ni un fidèle autoportrait ». Parce qu'il n'est écrit que pour soi et qu'il passe sous silence bon nombre d'informations contextuelles évidentes pour l'auteur, un journal intime est dès lors bien plus difficilement lisible qu'un billet de blog (Lejeune, 2000, pp. 30-33).

D'autre part, cette notion "d'extimité ", intègre plus fortement le caractère central de l'interactivité, des réactions des internautes dans le contenu des sites.

Et ce d'autant plus que de nombreux blogueurs interrogés (voir présentation de la méthodologie ci-après) se réapproprient le néologisme de blog extime plutôt que celui de blog personnel, comme pour revendiquer la démarche publique, collective de leur pratique :

« Nina : Un blog, c'est quoi ? En gros un espace perso où on peut partager ses passions ou raconter sa vie, entre autres [...]. Donc le but premier du blog, c'est de créer un journal extime où on partage tout ce que l'on veut partager. Sa vie amoureuse, sexuelle, professionnelle, ses pensées, ses joies et ses peines,

\footnotetext{
${ }^{3}$ L'emploi de l'origine de ce néologisme nourrit d'ailleurs beaucoup de discussions sur les blogs. Sur ce thème et ce terme d'extimité initialement utilisé par Serge Tisseron pour décrire la multiplication des témoignages, confessions dans des émissions de plateaux télévisées, voir Tisseron, 2001.
}

tic\&société - 2(1), 2008 
Les blogs « extimes » : analyse sociologique de l'interactivité des blogs

ses délires et tout ça. [...].. Si on est fragile, qu'on ne supporte pas les critiques et les jugements, un journal intime sera bien plus indiqué. Parce que quand on exhibe sa vie, forcément, celle-ci est analysée et jugée, pas forcément méchamment mais voilà ».

Plus significativement encore, l'interaction avec les lecteurs, l'attente de leurs commentaires, de leurs conseils, font partie intégrante des motivations des blogueurs :

« Juliette : [..] J'aime écrire pour être lue, pour partager avec les gens, réagir à leurs commentaires, alors qu'un journal intime est un exercice solitaire qui ne m'intéresse pas [...] Le blog était avant tout pour moi une façon de m'exprimer, d'entrer en contact avec les gens ».

\subsection{Les entretiens compréhensifs épistolaires}

L'analyse des blogs extimes sous l'angle de l'interactivité mêle alors deux objectifs :

1 - Le premier objectif de cet article est de mieux comprendre les motivations initiales des blogueurs extimes mais surtout le rôle joué par les réactions des lecteurs dans l'évolution de ces motivations. Ces réactions jouentelles dans les thématiques développées? Favorisent-elles un caractère consensuel ou à l'inverse polémique des discours tenus ?

2 - Le second est plus fondamentalement d'expliquer la perception globalement positive qu'ont les blogueurs des réactions des internautes, une satisfaction centrale si l'on veut expliquer pourquoi cette pratique compte autant de « pratiquants ».

Parce qu'il s'agit de comprendre, puis d'analyser, l'interprétation subjective que ces individus ont de leurs pratiques, la méthode de l'entretien compréhensif est ici pertinente.

L'entretien compréhensif a pour avantage de recueillir des données sur l'expérience vécue et de pénétrer le système de représentation des personnes, d'en dessiner les lignes de force et de pointer les contradictions avec les discours préalablement établis. Deux postulats sous-tendent cette démarche : 1. les pratiques ont une épaisseur, elles sont complexes et structurées ; 2 . le sujet n'a pas toujours raison, mais il a ses raisons, qu'il s'agit de découvrir, de connaître, de comprendre et pas de juger hâtivement, ni de réduire à quelques variables-clefs (Kaufmann, 1996, pp. 48-50). Dès lors, les personnes 
interrogées constituent moins un échantillon a priori représentatif qu'une sélection raisonnée de sources d'information. Ainsi, 20 entretiens approfondis de blogueurs francophones (France, Suisse, Canada) ayant entre 18 et 58 ans $^{4}$, dont $2 / 3$ de femmes et $1 / 3$ d'hommes, de divers milieux sociaux, ont été réalisés entre septembre et décembre 2007. La sélection s'est avant tout faite sur le type de blogs publiés plutôt que sur l'identité des blogueurs (hormis le souci d'une relative variété des profils des blogueurs), chacun de ces blogs parlant pour l'essentiel de la vie au quotidien de leur auteur et non de l'actualité politique, littéraire ou d'une passion. Enfin, les noms des blogueurs cités sont ceux que ces derniers auront utilisés pour se "définir " au cours de ces entretiens par mails (selon les cas, il s'agit parfois de leur vrai prénom, parfois de leur pseudonyme du blog, tous les blogueurs « extimes » n'ayant cependant pas de pseudonyme).

Or, malgré ses inconvénients (pas de panel représentatif, pas de croisement de variables extérieures - âge, statut, etc. - permettant de recontextualiser les données obtenues ${ }^{5}$ : Cardon, 1996, pp. 177-179), la richesse des enseignements obtenus par ce type d'entretien emphatique approfondi est significative. Ce type de recueil de données permet de comprendre ce que le cyberespace change à la pratique de construction d'un discours " médiatique » dans le cas de ces nouveaux acteurs; puis d'évaluer quelles normes sur les comportements jugés cybermédiatiquement "acceptables " "valorisants " ou au contraire " condamnables ", émergent.

Parce qu'il s'agit de comprendre l'impact de l'interactivité des blogs sur le contenu et la démarche des blogueurs, il n'est pas possible de s'en tenir à l'analyse des commentaires publiés en ligne (de surcroît après "modération » et donc sélection). Car une bonne part des réactions les plus directes, les plus touchantes, les plus engagées, les plus intimes et donc les plus susceptibles de

\footnotetext{
${ }^{4}$ Les blogs d'adolescents aux logiques, aux motivations et aux contenus souvent différents des blogs extimes analysés (plus de photos, moins de textes; une volonté affichée de rester entre-soi et de prolonger en ligne les conversations avec ses copains, etc.) n'ont pas été intégrés dans cette analyse. Pour une analyse de ce type de blog, voir la recherche de Trédan, 2005.

${ }^{5} \mathrm{Ce}$ panel raisonné ne permet ainsi pas de tester statistiquement de manière élargie l'hypothèse d'une corrélation entre type de réponse des enquêtés et variable de genre (homme/femme ?), de catégorie sociale (niveau de diplôme, rapport à l'écriture ?), etc. Le fait que plusieurs interviewés accordent par exemple une importance notable à la possibilité de mettre en ligne des informations, des expériences biographiques personnelles dont ils n'ont jamais osé parler à leur entourage afin de bénéficier des avis, conseils, encouragements de leurs "amis lecteurs" ne peut ainsi être directement expliqué par des variables extérieures dans la mesure où ces échantillons ne sont pas nécessairement construits sur des critères de représentativité fondés sur des hypothèses sociologiques initiales.
} 
Les blogs « extimes » : analyse sociologique de l'interactivité des blogs

jouer sur les motivations et les billets des blogueurs sont échangées par correspondance privée, et donc en dehors du blog.

Véronique: "Mes lectrices amies sont des mamans pour la plupart. Certaines ont bifurqué en privé car elles ont vite compris (plus vite que moi !!), que leur voisin, leur patron, leur belle-mère (!!!) pouvaient tomber sur ce blog et lire les commentaires... chose qu'elles ne veulent pas...».

Autrement dit, faire une analyse de l'interactivité des blogs à partir de leur seul contenu en ligne ne donnerait qu'un aperçu incomplet de l'impact des réactions des internautes sur les billets publiés.

Cette méthodologie est d'autant plus intéressante ici qu'elle peut se faire via des entretiens épistolaires approfondis. Le blog est l'une des activités sociales pour lesquelles l'entretien à distance, par mail interposé, respecte parfaitement la consigne selon laquelle il est préférable de mener les entretiens dans les lieux habituels de l'activité étudiée - l'enseignant dans sa classe, le sportif dans son gymnase... et le blogueur devant son clavier - car c'est dans son contexte quotidien que l'acteur social se remémore le plus facilement, le plus concrètement la somme des actions quotidiennes qui définissent le mieux son comportement, etc. (Lahire, 2005, pp. 157-160). Peut-être parce que cette démarche fait écho à l'intérêt que beaucoup de ces acteurs sociaux/blogueurs accordent à l'intérêt d'un positionnement réflexif sur leurs pratiques ou encore parce que ces entretiens passent par le biais de l'écriture qui est une forme de communication qu'ils valorisent quasi-unanimement, en tout cas les entretiens les plus creusés ont pris la forme de plusieurs dizaines de pages de conversations épistolaires approfondies ${ }^{6}$.

\section{Quatre formes valorisantes d'interactivité blogueurs/internautes}

Un des éléments communs à tous les blogs - extimes ou non - concerne la visibilité accordée à l'audience : compteur de pages lues, outils gratuits pour localiser géographiquement (villes) les dernières visites effectuées ${ }^{7}$, etc. La

\footnotetext{
${ }^{6}$ Voici brièvement le déroulement de ces entretiens: sur l'exemple de la dernière enquête de Jean-Claude Kaufmann (2007), exemple dont cette recherche s'est méthodologiquement inspirée, le courrier envoyé aux blogueurs incluait le lien suivant: « appel à « témoignage », hébergé par l'IUT Vichy de l'université de Clermont-Ferrand II, http://www.iutvichy.univ-bpclermont.fr/sr/ (présentation des travaux, de l'objectif de l'enquête, de l'auteur, hébergeur universitaire).

7 Par exemple, "Webstats est une page web d'outils analytiques gratuits vous permettant de recenser le trafic de votre site Internet et donc ainsi de porter un jugement sur le comportement de vos visiteurs ». Les rubriques proposées sont les suivantes : résumé, pronostics pour aujourd'hui,
} 
situation est paradoxale : alors même que le blog est une activité de loisirs, parfois une passion, en tout cas une activité non-professionnelle et donc en dehors de toute obligation de rentabilisation financière, les plates-formes placent les objectifs d'audience au centre des outils proposés et des objectifs des blogueurs.

Cette reconnaissance chiffrée est bien sûr un moyen pour les plates-formes de blogs d'offrir une motivation directe à leurs "membres", une raison supplémentaire les incitant à actualiser leurs blogs, à améliorer les référencements, augmenter les visites et - dans le cas d'hébergeurs gratuits - à accroître la publicité des bannières publicitaires. Mais l'audience constitue-t-elle une explication en elle-même suffisante des motivations des uns et des autres et de ce qui se joue dans cette construction interactive d'une identité personnelle? En fait, l'impact de cette audience dépend à la fois des motivations initiales des blogueurs et des raisons qui les poussent à continuer la publication de billets. Elle ne tient pas à une seule - et indéterminée - logique d'audience.

L'engouement que cette nouvelle activité suscite fait ainsi ressortir quatre types de motivations. Des motivations qui révèlent autant d'attentes d'expressions individuelles insuffisamment satisfaites jusque-là, à la fois par les autres médias (ou dans d'autres conditions, voir Mehl, 1996) et technologies de communication (SMS) et dans leur vie quotidienne: témoigner, dévoiler sa personnalité (éventuellement créative), donner son avis, écrire (le fait de tisser de nouveaux liens amicaux, constat récurrent de ces entretiens, ne sera pas traité ici pour deux raisons : faute de place et parce que si ces liens engendrent une nouvelle motivation d'écriture réelle et parfois imprévue, ces interactions ont une influence ressortant d'un autre registre de celle des quatre autres facteurs analysés ici ; voir par exemple Cardon et Delaunay-Teterel, 2006, pp. 1-60). Or, quelle que soit la motivation dominante du blogueur, ce serait une erreur de considérer que les réactions de l'internaute jouent un rôle minoritaire (Nardi, Schiano et Gumbrecht, 2004, pp. 224-230). Elles ont au contraire une influence - variable selon les registres - parfois indirecte mais toujours déterminante dans l'évolution dynamique du blog (tableau 1).

répartition géographique des derniers visiteurs, pages vues par jour (sur un mois), pays. Voir : http://www webstats. motigo.com/.

tic\&société - 2(1), 2008 
Les blogs « extimes » : analyse sociologique de l'interactivité des blogs

Tableau 1 - Types d'identités mises en avant et formes d'interactivités correspondantes

\begin{tabular}{|c|c|c|}
\hline $\begin{array}{c}\text { Motivation du } \\
\text { blogueur }\end{array}$ & $\begin{array}{l}\text { Identité } \\
\text { valorisée }\end{array}$ & $\begin{array}{l}\text { Interactivité : } \\
\text { caractère valorisant } \\
\text { des réactions des } \\
\text { internautes }\end{array}$ \\
\hline $\begin{array}{c}\text { Revendiquer sa } \\
\text { vraie nature }\end{array}$ & $\begin{array}{c}\text { Comme individu } \\
\text { ayant une } \\
\text { personnalité } \\
\text { propre }\end{array}$ & $\begin{array}{c}\text { Reconnaissance de } \\
\text { sa personnalité } \\
\text { « authentique » }\end{array}$ \\
\hline $\begin{array}{c}\text { Faire valider sa } \\
\text { vie }\end{array}$ & $\begin{array}{c}\text { Comme témoin } \\
\text { d'un mode de } \\
\text { vie }\end{array}$ & $\begin{array}{c}\text { Réconforter et être } \\
\text { réconforté, } \\
\text { s'encourager } \\
\text { mutuellement }\end{array}$ \\
\hline $\begin{array}{c}\text { Donner son } \\
\text { avis, juger } \\
\text { réfléchir }\end{array}$ & $\begin{array}{c}\text { Comme } \\
\text { «essayiste » }\end{array}$ & $\begin{array}{c}\text { Débattre, échanger, } \\
\text { convaincre de la } \\
\text { gravité } \\
\text { d'un sujet }\end{array}$ \\
\hline \hline Écrire & Comme & $\begin{array}{c}\text { La fidélité du public } \\
\text { attendant le billet } \\
\text { suivant }\end{array}$ \\
\hline
\end{tabular}

Bien sûr, le succès des blogs extimes tient alors en partie à leur capacité à prendre en charge des besoins d'expression variés dans la population. Mais il tient aussi à une seconde caractéristique qui ressort de ces pratiques: le caractère jugé "valorisant », «enrichissant », «épanouissant », parfois "nécessaire » même de publier un blog extime et de s'y connecter en permanence.

Frédéric : «Aujourd'hui, j'ai du mal à décrocher, partir en week-end ou à l'étranger et ne pas avoir Internet pour livrer mes impressions ».

Comment expliquer que le mode de construction et de valorisation de l'identité via un blog personnel soit quasi-unanimement perçu comme 
valorisant? Comment expliquer surtout que les réactions reçues soient systématiquement considérées comme positives?

\title{
2.1 - Revendiquer sa « vraie » personnalité »: le blog extime comme espace de reconnaissance
}

La première motivation des blogueurs extimes est - logiquement d'exprimer sa « vraie personnalité ».

\begin{abstract}
Paloma : «Et là, je me suis rendue compte que peu me connaissaient réellement. Nous passons finalement notre vie avec des masques, plus ou moins épais à remplir le rôle qui nous est dévolu, ou dans lequel nous avons bien voulu nous glisser. Et nous passons notre vie à idéaliser les gens que nous aimons, nous voilant ainsi plus ou moins la face sur des aspects de leur caractère qui, potentiellement, nous révulseraient chez d'autres ».
\end{abstract}

En soi, l'idée que la présentation d'une identité soit en permanence remodelée par le regard de l'autre n'est pas nouvelle. Mais en quoi l'intégration des internautes dans la phase d'écriture de cette autobiographie sur cette expérience personnelle, en quoi le dispositif des blogs, en modifie-t-il la teneur ? S'agit-il d'exposer ou de faire valider sa vie ${ }^{8}$, de donner un témoignage sans en attendre de retour ou, comme le pense Annabelle Klein, de solliciter le regard de l'autre dans la construction de son identité (2001)?

Le premier type d'interprétation et d'explication de la croissance de la blogosphère personnelle part de l'idée - communément partagée - selon laquelle "le modèle dominant de l'identité au sein des sociétés modernes avancées est l'affirmation d'un "moi d'abord" sous la double modalité de l'humain qui se trouve en chacun d'entre nous (face publique avec la revendication des droits de l'homme) et de l'originalité (face privée avec la revendication de l'identité cachée) 》 (Singly, 2003, p. 82).

Cette importance est d'abord l'un des multiples révélateurs et l'une des diverses conséquences de la valeur aujourd'hui accordée à l'expression d'une

\footnotetext{
${ }^{8}$ Cette hypothèse est exposée par Laurence Allard et Frédéric Vandenberghe qui constatent que l'on "repère de nombreuses traces paratextuelles de ratifications publiques de cette "identité écran" dans les pages étudiées : liens vers une adresse de courriel, possibilité de laisser des commentaires dans un livre d'or », etc. (2003, p. 212).
} 
Les blogs « extimes » : analyse sociologique de l'interactivité des blogs

nouvelle individualité. En deçà des différences existant entre les multiples formes d'individualismes possibles (comme autonomie d'expression, comme réalisation de soi, comme indépendance émotionnelle, etc. : Loose, 2000, pp. 59-107), notre société se caractérise, sur un temps historique long, par l'avènement de l'individu. "Valeur suprême, l'individu est légitime dans la diversité de ses envies, de ses goûts, de ses pratiques [...] " (Prost, 2002, pp. 23-27). Une émergence qui tiendrait à une convergence et à l'interaction de trois choses: l'individualisme comme tendance de très longue durée, la rationalisation d'une société qui s'appuie sur une rationalité instrumentale de plus en plus perfectionnée et la différenciation sociale au sens de détraditionalisation des formes collectives d'identité (montée de la globalisation, disjonction entre croyance et socialisation, crise des identités professionnelles, etc. (Dubar, 2000 ; Hervieu-Léger, 1996 ; pour une synthèse voir Ascher, 2005, pp. 11-34).

Ainsi, de moins en moins d'acteurs sociaux se définissent subjectivement à partir de critères de classes sociales ; ou plutôt ils préfèrent d'eux-mêmes se situer de plus en plus nombreux dans la vaste et indéterminée «classe moyenne " (Boltanski, 1982, pp. 63-65). Dans le même ordre d'idée, le constat du déclin des normes traditionnelles depuis la Deuxième Guerre mondiale est aujourd'hui largement partagé (Singly, 2000, pp. 17-18). Cette tendance est marquée par exemple par une plus grande permissivité sexuelle, par la remise en cause partielle de l'institution familiale élargie, par la contractualisation des liens de mariage (Théry, 2001), etc. Dès lors, les individus sont, au moins subjectivement en charge de la définition d'une identité protéiforme (Halpern, 2004).

De fait, le degré de réalité de cette autonomie individuelle fait aujourd'hui débat entre sociologues. Faut-il considérer malgré tout incontournable ou au contraire désormais secondaire le milieu social, les catégories socioprofessionnelles dans les trajectoires individuelles ou dans la hiérarchisation des définitions des identités individuelles (Mendras, 1994, pp. 29-95 ; Chauvel, 2001, pp. 315-359 ; Lahire, 2005, pp. 261-305 ; etc.) ? Faut-il davantage considérer l'importance accrue de facteurs culturels, d'âge, etc. accréditant les thèses d'identités individuelles plus éclatées, voire plus librement déterminées?

Reste que, quelle que soit la réalité de ce sentiment d'autonomie concernant la «libre détermination» de l'identité de chacun, ce travail vise à mesurer de quelle façon la pratique du blog concourt à renforcer l'idée d'une construction individuelle de son identité personnelle. Une idée renforcée par la pratique des blogs extimes pour plusieurs raisons. 


\subsubsection{Une publicisation maîtrisée de son image en ligne}

De fait, la pratique du blog extime permet d'exposer publiquement sa vie, ses idées, ses ennuis, son identité privée, voire intime tout en contrôlant autant que possible les modalités de cette exposition. Sur un blog, le blogueur choisit seul le terrain sur lequel il souhaite aller. Or, cette liberté tranche non seulement avec la situation la plus courante dans les médias généralistes dans lesquels les journalistes jouent un rôle de médiateur/prescripteur implicite d'identités médiatiquement légitimes incontournables (Rouquette, 2001; Macé, 2006), mais aussi avec le cadre de vie ordinaire. En général, la casquette identitaire de la personne dépend du lieu sur lequel elle se trouve. Dans son usine, on est en droit d'attendre d'elle qu'elle se présente et se comporte prioritairement selon son identité professionnelle de cadre ou d'ouvrier, dans une école comme parent d'élève, etc. (Singly, 2000, p. 90). Dans le monde virtuel des blogs, non seulement chaque blogueur a un degré de contrôle plus élevé du terrain identitaire sur lequel il veut aller mais il le fait avec un niveau de maîtrise supérieur (Burnett et Marshall, 2003, pp. 270-273; Trédan, 2005, p. 6). Ou plutôt, comme le montrent plus précisément encore les entretiens effectués avec les blogueurs, le blog semble au tout début de leur activité réunir les conditions pour se dévoiler publiquement et sans risque. D'ailleurs, la recherche de John Bargh et Katelin Mc Kenna, consacrée aux aspects stigmatisés de l'identité sexuelle d'usagers des forums de cybersexe a bien montré qu'Internet est perçu comme un outil permettant de trouver - sans risque et bien plus facilement que dans leur vie quotidienne - des gens partageant les mêmes préférences (2004, pp. 578-585).

Le blogueur a donc la latitude de celui qui non seulement décide des règles du jeu (de présentation de soi et du dispositif de communication ici) mais qui a de surcroît la possibilité d'adapter ces règles en connaissance de cause, au fur et à mesure des réactions reçues. II peut se relire, changer une expression avant de la publier, modifier un titre, changer une bannière, mettre en avant ou enlever un billet. Or, dans la vie quotidienne, ce type de situation d'interaction entre les acteurs sociaux, n'est, d'une part, le plus souvent pas aussi déséquilibré (hormis lorsqu'on reçoit chez soi ou encore dans le cas de rapports hiérarchiques dans un lieu professionnel).

D'autre part, il leur est, sur la durée, plus facile de contrôler leur image extérieure, contrairement aux interactions en face-à-face dans lesquelles les acteurs sociaux échangent deux types d'informations : celles qu'ils donnent et celles qui leur échappent. D'après les observations de chercheurs du « collège invisible » de Palo Alto, les gens laissent échapper malgré eux beaucoup d'informations à travers leur posture, leur regard, leur voix, etc. (Birdwihistell, 1981, pp. 160-172). L'analyse des rituels sociaux d'Erving Goffman s'appuie également sur une observation fine et rigoureuse des interactions quotidiennes.

tic\&société - 2(1), 2008 
Les blogs « extimes » : analyse sociologique de l'interactivité des blogs

Pour en rendre compte, il utilise une métaphore dramaturgique : la vie sociale peut être comparée à une scène où des acteurs interprètent des rôles en accord avec la situation (Quéré, 1989, p. 54). Cette métaphore n'implique nullement que la vie sociale soit factice et que les acteurs soient nécessairement conscients de « jouer ». Au contraire, ils sont le plus souvent fortement impliqués dans le jeu et le ressentent comme spontané (Winkin, 2001, pp. 91102). Reste que, dans une rencontre réelle, ce sont les informations qui échappent aux gens qui sont les plus recherchées, et non celles qu'ils donnent volontairement (Goffman, 1973).

Or, cet écart entre les interactions par " posts " ou commentaires interposés et les interactions en face-à-face $a$, contrairement aux relations de séduction à distance (Illouz, 2006, pp. 250-268), bien plus d'avantages que d'inconvénients, tout au moins pour les blogueurs. Cette "réduction » de l'image de soi en mots implique que celle-ci est nécessairement consciente, voulue et donc beaucoup plus maîtrisée. Ils s'expriment autant qu'ils le souhaitent dans les limites qu'ils se sont - au fur et à mesure - fixées; libre à eux ensuite d'accepter ou de refuser d'aller plus loin dans les échanges par mails privés, libre à eux d'engager des relations directes plus contrôlées et moins approfondies ou d'en rester à ce niveau de relation potentiellement déjà satisfaisant.

Significativement, cette satisfaction d'en rester à ce niveau de présentation de soi n'est pas partagée par tous leurs lecteurs.

Nina : « Je suis étonnée à chaque fois que je reçois des messages de la part d'internautes qui ont cherché et trouvé des infos sur moi [..]. Je ne comprends pas trop ce besoin de chercher mais bon, c'est le jeu. Mais je me sens un peu touchée dans mon intimité et ça, je n'aime pas trop. J'ai posé les limites de l'anonymat, j'ai choisi un pseudo, j'ai même changé le nom de mon chat donc j'aimerais qu'on me laisse tranquille de ce côté-là ».

Comme si le caractère écrit de cette présentation interactive provoquait chez ces derniers un sentiment de frustration, dans l'impossibilité qu'ils sont de se construire eux-mêmes une image totale de la personne au récit de laquelle ils se sont progressivement attachés. Reste que, du point de vue des blogueurs, le caractère a minima écrit et contrôlé de cette relation est, en soi, satisfaisant. 


\title{
2.1.2 Une identité choisie
}

Ce niveau de présentation est d'autant plus suffisant que le blogueur a, pour sa part, une grande liberté : celle de pouvoir s'exprimer sur les sujets qu'il souhaite. Fait révélateur, plusieurs des blogueurs interrogés marquent leur surprise, voire leur désappointement devant les réactions d'internautes se focalisant sur un seul trait de leur personnalité. Ce type de réaction ne doit pas seulement s'expliquer par le fait que cette lecture va à l'encontre de l'image multifacette que chacun se construit progressivement de lui-même dès sa petite enfance (Lecuyer, 1994) mais plus frontalement encore parce qu'elle va à l'encontre de leur identité volontairement projetée sur le web. Ainsi de Nina :

\begin{abstract}
«...les personnes retiennent surtout le côté hypra sexué de ce blog alors que je me suis beaucoup calmée sur les récits sexy. [...]. Aujourd'hui, je ne parle plus de ça [...] et pourtant, ça reste. Et c'est pénible, des fois parce que [...] ce serait bien de retenir aussi ce que je dis dans d'autres domaines ».
\end{abstract}

Si comme l'ont montré de multiples études de réception, le texte lu et reçu ne correspond jamais au sens voulu par l'auteur (Dayan, 1992, pp. 153-154) - de multiples facteurs jouant sur les distorsions occasionnées entre l'encodage et le décodage de tout contenu médiatique (Pasquier, 1998, pp. 213-233 ; Esquenazi, 2003), blogs extimes compris - le fait que cette distorsion se produise sur un «texte » qui met en jeu l'image et l'identité de l'auteur de ce "texte» explique ici l'importance accordée à cette «distorsion »; un peu comme si le lecteur prenait la « liberté » de lire l'histoire de sa vie autrement que celle l'on privilégie soi-même. En ce sens d'ailleurs, l'interactivité joue un double rôle d'ajustement. Si les blogueurs tiennent compte des commentaires des lecteurs dans l'écriture de leur blog, la réciproque est tout aussi envisageable, le blogueur pouvant toujours tenter de « recadrer » les lecteurs sur « l'objet réel de ce blog ».

\subsubsection{Une image positive de soi}

On ne peut mesurer le caractère central du caractère valorisant des blogs extimes sans évaluer à quel point la majorité des blogueurs interrogés attachent eux-mêmes de la valeur à se montrer sous leur « meilleur jour » :

«Le blog n'est qu'une vitrine, une belle, une

embellie... de nous-mêmes... du moins pour les 
Les blogs « extimes » : analyse sociologique de l'interactivité des blogs

blogueurs qui ne me parlent pas en privé... après, quand on passe la barrière du blog, parce l'amitié est installée, on peut passer à des confidences... c'est protégé, c'est entre nous...[...] » (Françoise).

Ce constat s'explique largement par une tendance commune qui consiste à se présenter de manière générale par une image positive, surtout face à des inconnus. Véronique explique :

«Pour en revenir au fait de ne pas écrire quand ça ne va pas, il y a aussi un refus à se montrer sous un mauvais jour, quand le spleen est là... c'est un peu comme dans la vraie vie, on voudrait toujours montrer le meilleur de soi-même... ne pas décevoir... sauf que dans la vraie vie, on ne peut pas tricher... dans un blog, on est protégé, il n'y a pas la confrontation physique. Donc on peut être mal peignée derrière son écran, on s'en tape, personne ne nous voit !».

Comme le montrent les études sur la manière dont chacun reconstruit en permanence l'histoire de sa vie, tout mode de (re)construction d'une identité se produit fondamentalement sur un mécanisme toujours positif. Cet exercice permanent de mise à jour autobiographique fait apparaître deux tendances complémentaires. La première est qu'il passe par l'occultation des phénomènes les plus douloureux de l'histoire personnelle. La seconde est qu'il est caractérisé par une nette tendance à l'auto-valorisation, en particulier par une rationalisation a posteriori des événements positifs (Martinot, 2001, pp. 484-485). Si la représentation d'une identité positive de son existence et de sa personnalité constitue l'une des conditions d'un meilleur épanouissement personnel, on comprend alors tout l'intérêt que revêt chaque pratique favorisant cette valorisation de soi, d'abord à ses propres yeux.

Au total, de multiples facteurs convergent pour qu'un blog extime favorise la mise en ligne d'une image de soi conforme à ce que l'on souhaite. Cette authenticité, assumée parfois avec la crainte des réactions mais finalement engagée à moindre coût, renforce en tout cas le sentiment - vrai ou exagéré de maîtriser sa vie et de pouvoir en assumer les choix quel que soit le domaine : religieux, politique, culturel, etc. De ce point de vue, le développement des blogs n'est pas seulement l'expression d'une idéologie (l'individualisme comme expression de son autonomie), il en favorise la visibilité, sinon l'expansion. 


\subsection{Témoigner : le blog comme espace de réconfort}

Parallèlement à l'opportunité de pouvoir revendiquer sa personnalité, le blog personnel est souvent interprété comme le moyen de «témoigner sur sa vie », c'est-à-dire sur son quotidien, sur un changement de vie, sur ses ennuis, sur ses expériences, etc. Les influences envisageables des internautes sont multiples.

Bien des blogueurs interrogés font remonter la décision de publier leur vie en ligne à un événement déclencheur marquant, probablement parce que la décision de parler publiquement de sa vie, au-delà de toute considération narcissique, engage bien plus fortement la personnalité de son auteur que les blogs politiques, culturels ou de loisirs. Ce peut être un changement de ville, une nouvelle étape dans sa vie comme un événement douloureux.

Or, à deux limites près - le choix de la plate-forme jouant, sur le type de public touché selon son caractère commercial, généraliste ou spécialisé (pour ne pas dire « affinitaire par centre d'intérêt ») et l'importance de l'audience du blog (le nombre d'internautes «indélicats » augmentant proportionnellement avec le succès du site) - les réactions interactives des internautes (sur soi ou ce que l'on leur conseille de faire) sont la plupart du temps perçues comme encourageantes.

Capucine : «[avant] j'étais hébergée chez Skyblog, [...] le problème, selon moi, est qu'elle dépend de la radio Skyrock et que, du coup, elle amène un flot assez incalculable de commentaires négatifs ou qui ne servent carrément à rien. [...]. Moi, je sortais un peu du lot et publiais des chroniques féminines. Comme j'étais pas mal lue, j'avais mon lot de : "Lâche tes commentaires" et autres publicités que je supprimais automatiquement. Parce que sur Skyblog, c'est monnaie courante ».

Comme l'exprime par exemple Véronique :

« Je me trouve toutefois chanceuse, je n'ai jamais eu de commentaires désobligeants. Les internautes me félicitent beaucoup en moyenne pour la mise à plat de mon quotidien, la facilité à avouer que je suis humaine, que je craque, que je traverse la maladie de mes enfants avec acharnement et grand désespoir aussi ». 
Les blogs « extimes » : analyse sociologique de l'interactivité des blogs

Comment expliquer le caractère aussi "positif » de cette seconde forme d'interactivité ? Plusieurs facteurs entrent en jeu.

Le premier est que la palette des réactions jugées positives est très étendue. C'est parce que les attentes des blogueurs ne sont pas toujours bien définies, parce que le dispositif leur permet également d'aborder un panel très vaste de sujets, qu'un grand nombre de réactions rentre dans la catégorie des " commentaires positifs". Autrement dit, limiter le feedback des blogs extimes à la validation de l'intérêt de sa propre vie serait ici réducteur. L'interactivité d'un blog extime ne joue pas qu'un rôle de rappel des normes et donc de validation de son parcours individuel en fonction des normes collectives. Loin de passer nécessairement par une validation des choix personnels des blogueurs ou par une admiration affichée, ce besoin de reconnaissance se traduit parfois " simplement » par de la compassion, de la compréhension (Cheung, 2004, p. 61).

Le second est que, corollairement ce type de billet intime, dévoilant aux autres ses doutes, ses angoisses, constitue le type même de contenu qui appelle un grand nombre de commentaires positifs. L'encouragement anonyme suffisant, le degré d'engagement demandé à l'internaute n'est pas rédhibitoire.

Le troisième est qu'une partie des lecteurs sont d'autant plus facilement touchés qu'ils ont eux-mêmes connu des expériences similaires. Internet facilite la constitution d'espaces publics médiatiques segmentés dans lesquels chaque internaute construit son panel de sites et de blogs préférés en fonction de ses propres centres d'intérêts. Si un internaute "tombe » éventuellement sur un blog par hasard (le fameux effet serendip : au hasard des liens), il y revient nécessairement par choix.

Autrement dit, l'intensité de l'interactivité entre blogueurs extimes et lecteurs est d'autant plus probable que la thématique du blog est segmentée et donc l'audience culturellement, affinitairement comparable à celle du blogueur (chômage, emploi précaire, rupture amoureuse, maladie infantile grave, etc.). En comprenant, encourageant alors le "même que soi " c'est sans doute un peu de soi qu'on comprend et qu'on encourage.

Dans tous les cas, cette interprétation de l'interactivité implique de considérer le blog comme une forme d'activité sociale à part entière (et non comme une stricte activité de loisir ou une activité « littéraire »). Le caractère "social » de cette activité d'écriture/lecture apparaît par ailleurs très nettement dans la réciprocité des échanges, les blogueurs se citant par blogrolls interposés mais aussi se lisant, se commentant, voire s'encourageant par cercles (concentriques) d'affinités. 


\subsection{Donner son avis, convaincre : s'engager, affirmer ses choix}

Par définition, la mise en ligne d'un blog extime a pour motivation initiale de parler de sa vie. Mais peu refusent de parler de tout, y compris de l'actualité. Un blogueur extime se présente aussi sous les traits d'un « commentateur public de l'actualité » sans l'avoir nécessairement prémédité. Or, l'interactivité d'un blog change complètement la manière dont les blogueurs abordent cette activité quotidienne qui consiste à affirmer ses choix, sa personnalité en échangeant avec d'autres son avis sur les sujets du jour. L'influence des lecteurs sur les idées sociales, politiques, culturelles de l'auteur des blogs extimes se marque par son caractère inattendu, voire imprévisible.

Cette influence est double. Un blog extime incite d'une part fortement ses auteurs à s'engager sur des sujets connexes à ses billets. II les oblige d'autre part à défendre et donc à affermir leurs positions: "C'est presque un phénomène de "ricochet" ... Bon nombre de billets commencent par "J'ai lu ceci sur le blog de... ce qui m'a donné envie de vous parler de cela....".... C'est assez fréquent. La perte d'inspiration d'un blogueur peut se réveiller grâce au billet d'un autre... et ouvrir encore un débat... c'est magique.... c'est le principe !» (Françoise). Donner la possibilité de s'exprimer dans l'espace médiatique oblige à justifier, à défendre, à assumer ses propos présents et passés, à se construire une image cohérente de soi-même, de ses témoignages ou de ses idées.

On le voit, l'usage du blog extime mêle deux facettes : d'un côté il facilite le dévoilement public de témoignages privés, de l'autre il oblige ces témoins à assumer publiquement leurs choix, et donc - selon la tournure des débats - à les expliquer, les motiver, les justifier. Or, sur un plan plus général, selon les contextes, les types de raisonnement adoptés par les acteurs sociaux diffèrent: raisonnement de type «scientifique » (agir en termes de connaissances, balayer l'ensemble des hypothèses disponibles, accepter le doute), de type " pragmatique » (aboutir à une décision dans les meilleurs délais quitte à se fier à son intuition, son expérience) (Gardair, 2007, pp. 40-46). Dans le contexte particulier des blogs, l'un des impacts des forums est alors d'inciter les blogueurs à adopter un mode de raisonnement sur soi, sur ses comportements, réfléchi, argumenté, d'une certaine façon rationalisé.

\subsection{L'importance de l'écriture : le blog extime comme auteur}

Du point de vue des blogueurs, le caractère écrit du dispositif est central. II ne fait pas que faciliter une présentation contrôlée et cohérente de soi, il leur donne une nouvelle position : celle « d'auteur». 
Les blogs « extimes » : analyse sociologique de l'interactivité des blogs

Sur un plan strictement littéraire ou social, on peut toujours rappeler que la légitimité d'un blogueur à succès n'a - jusqu'à aujourd'hui - rien de comparable à celle d'un auteur de roman publié par une maison d'édition. D'ailleurs le fait que les blogueurs eux-mêmes considèrent comme une forme de reconnaissance ultime la publication papier de leur blog rappelle la somme des différences qui existent socialement entre un auteur de roman et un «presqueauteur » de blog (pas de droit d'auteur, de séance de signature, d'émission littéraire télévisée hebdomadaire, etc.).

\begin{abstract}
Nina: "Après, je crois que le critère de reconnaissance ultime a été d'avoir été contactée par deux maisons d'éditions pour éditer les blogs [...] ça montre que dans mon métier qu'est l'écrit, je suis bonne. Et je crois que je n'ai jamais eu une critique de qui que ce soit sur mon style ».
\end{abstract}

Mais pour qu'un acteur se sente légitime à endosser - au moins partiellement - une telle position, c'est-à-dire ici une compétence culturelle et intellectuelle associée à la position d'auteur, la reconnaissance publique n'est pas incontournable. À l'échelle interindividuelle adoptée ici, le plus important est que les blogueurs, incités qu'ils sont à publier aussi régulièrement que possible la suite de leurs "aventures quotidiennes" en raison de la forte visibilité donnée à l'audience des sites, soient considérés comme tels par une partie de leurs lecteurs.

Christine : «Quelquefois, je ne suis pas inspirée, je n'ai pas envie, j'ai autre chose à faire. Et bien, mes lecteurs les plus accros me rappellent à l'ordre! Je fais partie de leurs vies. Honnêtement, cela rajoute une petite pression supplémentaire : il faut rendre son "billet" tous les jours ».

Ils sont en position d'acteurs sociaux évalués en permanence, stimulés par la présence d'un auditoire régulier, qu'il ne faut surtout pas décevoir. L'importance qu'ils attribuent à cette reconnaissance se mesure alors à l'attachement qu'ils accordent à la qualité stylistique et presque littéraire de leur blog. Anaïs explique :

"C'est vrai qu'après, quand on voit des gens qui nous complimentent sur notre style, qui aimeraient être à 
notre place et savoir écrire comme nous, ça fait du bien ».

II y a bien sûr dans ce sentiment de reconnaissance le fait de se voir discerner des qualités et des savoir-faire proprement littéraires : que ce soit sur leur style, leur capacité à émouvoir, à savoir raconter des histoires. Une reconnaissance qui, d'ailleurs, joue un rôle - clairement assumé par plusieurs blogueurs - soit de compensation face à un manque de confiance en soi, soit de validation de compétences intellectuelles et même professionnelles (surtout pour ceux qui vivent une trajectoire professionnelle chaotique): productivité écrite, diversité des centres d'intérêts, sens du lecteur, connaissance de la blogosphère (indispensable pour être bien référencé), etc. (un sentiment d'ailleurs partiellement fondé puisque 3 des 20 blogueurs interrogés ont trouvé ou changé de travail grâce à leur site en ligne).

Mais une autre explication se mêle à celle-ci. Cette satisfaction dans le sentiment de reconnaissance de la qualité du travail littéraire accompli est d'autant plus grande que les dispositifs des plates-formes placent les blogueurs en situation d'évaluation permanente. Si, comme n'importe quel individu se sachant observé par d'autres (Zajonc et Hazel, 1982, pp.123-131, cités par Myers et Lamarche, 1991), les blogueurs se sentent stimulés et encouragés à produire des billets les plus intéressants possibles, il est aussi logique qu'ils retirent plus de satisfaction dans le « couronnement » des « efforts » consentis.

\section{Conclusion}

Finalement, non seulement le phénomène des blogs extimes répond à de multiples attentes potentielles, mais il le fait sous des formes jugées enrichissantes personnellement et socialement. Plus précisément encore, les différentes formes d'interactivité des blogs sont bien plus souvent une motivation supplémentaire qu'une contrainte décourageante, un attrait allant jusqu'à modifier parfois les objectifs initiaux des créateurs de sites.

Ainsi, coexistent pour les blogueurs de multiples raisons de trouver dans les réactions des internautes/lecteurs une forme de reconnaissance identitaire satisfaisante : que ce soit sur la personnalité, la vie, les qualités d'écrivain ou les idées formulées par le blogueur. S'ajoutent à cela un grand nombre de facteurs contextuels favorables à la constitution de liens sociaux affinitaires (possibilité d'évaluer facilement les ressemblances, les points communs). Et ce sans que pratiquement aucune caractéristique de la blogosphère ne vienne freiner cette tendance (dévoilement facilité par un sentiment de protection relative si l'on utilise un pseudonyme, possibilité de se désengager d'un clic de relations jugées «envahissantes» ou «pesantes»). Si le blog est perçu comme une 
Les blogs « extimes » : analyse sociologique de l'interactivité des blogs

activité si valorisante de présentation de soi, peut-être est-ce au fond parce que le niveau de satisfaction qu'on en retire est proportionnel au degré de sincérité initialement consenti? Et, dès lors, sur le fait que les caractéristiques des blogs en ligne facilitent effectivement une telle franchise, d'abord vis-à-vis de soimême et, secondairement, d'autrui ?

Ces résultats appellent ainsi d'autres interrogations concernant le caractère potentiellement fictif de l'identité électronique de ces blogueurs.

II peut apparaître comme a priori logique de suspecter parmi les blogueurs, la construction d'identités fictives. De telles «impostures identitaires » permettant alors soit de tester en ligne un comportement difficile à adopter au quotidien, soit de rendre sa vie plus mouvementée, plus riche, en un mot plus " captivante » à lire qu'elle ne l'est à vivre. De telles lectures des blogs extimes sont d'ailleurs a priori d'autant plus plausibles que plusieurs blogueurs ont effectivement été découverts en flagrant délit "d'invention identitaire " (Fosseux, 2001, pp.52-58). Or, à la lumière des résultats précédents, de telles pratiques ne sont-elles pas minoritaires ${ }^{9}$ ? Typique d'une explication en termes de recherche d'audience, ces interprétations n'occultent-elles pas une motivation centrale des blogueurs : avant d'être un travail d'écriture pour autrui, un blog extime n'est-il pas un travail de réflexion pour soi, sur son identité (quelle qu'elle soit) et sur la reconnaissance de celle-ci par autrui ? Quelle satisfaction personnelle (comme individu et non comme auteur) un blogueur pourrait-il retirer d'une telle reconnaissance fondée sur une identité imaginaire $^{10}$ ? On le voit, cette approche compréhensive des facteurs et des conditions qui encouragent cette pratique singulière - décider de raconter chaque jour à des millions d'inconnus (potentiels) ses péripéties quotidiennes, ses envies, ses colères, ses passions - est si riche qu'elle ouvre d'autres pistes d'analyse futures.

\footnotetext{
${ }^{9}$ Tentation d'ailleurs vérifiée chez un petit nombre de blogueurs interrogés, jusqu'à ce qu'euxmêmes trouvent moins d'intérêt personnel à broder la réalité qu'à la dépeindre telle qu'elle est pour "se faire du bien ». Nina : "La tentation pour un blogueur de faire du racolage pour avoir toujours plus de lecteurs est grande, il faut l'avouer. Voir le chiffre de lecteurs augmenter, c'est grisant, le voir redescendre, inquiétant : zut, je les ai vexés, je ne leur plais plus, au secours, que se passe-t-il ? Là, se pose une question fondamentale : blogue-je pour moi ou pour mon lectorat ? À quoi ça sert de s'exprimer si je ne suis pas écoutée ? Est-il risqué de parler de tel sujet, ne vais-je pas fâcher/choquer les gens? ".

${ }^{10}$ Thomas : "Je ne me considère pas spécialement engagé à tenir un témoignage authentique par contrat implicite avec le lecteur mais par contrat avec moi-même. Je fais ce blog avant tout pour moi, pour dire ce que je pense, pour passer un coup de gueule ou pour partager un coup de blues. Je ne mens pas parce que ça ne m'apporterait rien, tout simplement ».
} 


\section{Références bibliographiques}

ALLARD L. et VANDENDERGHE F., 2003, « Express yourself ! Les pages perso ", Réseaux, n¹17, pp. 190-219.

ASCHER F., 2005, La société hypermoderne, La Tour d'Aigues, Editions de l'Aube.

BARGH J. et MCKENNA K., 2004, « The internet and social life », 2004, Annual Revue of Psychology, n 055 , pp. 573-590.

BIRDWIHISTELL R., 1981 (1968), « Un exercice de kinésique et de linguistique : la scène de la cigarette ", dans La nouvelle communication, WINKIN Y. (dir.), pp. 159-190.

BOLTANSKI L., 1982, Les cadres. La formation d'un groupe social, Paris, Minuit.

BOURDELOIE H., 2000, « Des discours d'accompagnement aux usages des technologies culturelles ", <http:/www.creis.sgdg.org/colloques\%20creis/2001/is01_actes_colloque/bourde loie.htm>, dernière consultation septembre 2008.

BURNETT R. ET MARSHALL D., 2003, «The look of the web », Web Theory : an introduction, Londres, Routledge, pp. 81-104.

CARDON D., 1996, «L'entretien compréhensif : note de lecture », Réseaux, n 079 , pp. 177-179.

CARDON D., et DELAUNEY-TETEREL H., 2006, « La production de soi comme technique relationnelle », Réseaux, ${ }^{\circ}{ }^{\circ 138, ~ p p . ~ 15-71 . ~}$

CHAUVEL L., 2001, «Le retour des classes sociales ? ", Revue de l'OFCE, n ${ }^{\circ} 79$, pp. 315-359.

CHEUNG C., 2004, « Identity construction and self-presentation on personal homepages ", Web studies, Londres, Éditions Arnold, pp. 53-68.

DAYAN D., 1992, «Les mystères de la réception », Le Débat, n71, pp. 146162.

DUBAR C., 2000, La crise des identités. L'interprétation d'une mutation, Paris, Presses Universitaires de France.

ESQUENAZI J.-P., 2003, Sociologie des publics, Paris, La découverte.

FOSSEUX S., 2001, Les journaux personnels en ligne : le paradoxe des confidences publiques, Mémoire de Maîtrise, Paris II. 
Les blogs « extimes » : analyse sociologique de l'interactivité des blogs

GARDAIR E., 2008, " Heuristiques et biais ", Revue électronique de Psychologie Sociale, $\mathrm{n}^{\circ}$, pp. 35-46. <http://www.psychologiesociale.org/reps 1. php?article=5>, dernière consultation septembre 2008 .

GOFFMAN E., 1973 (1959), La mise en scène de la vie quotidienne : la présentation de soi, Paris, Éditions de Minuit.

HALPERN C., 2004, «Faut-il en finir avec l'identité », Identité(s). L'individu, le groupe, la société. Paris, Éditions Sciences humaines, pp. 14-17.

HERVIEU-LEGER D. et DAVIE G., 1996, Identités religieuses en Europe, Paris, La découverte.

ILLOUZ É., 2006, « Réseaux amoureux sur Internet », Réseaux, n¹38, pp. 244268.

KAUFMANN J.-C., 1996, L'entretien Compréhensif, Paris, Nathan.

KAUFMANN J.-C., 2007, Agacements : les petites guerres du couple, Paris, Armand Colin.

KLEIN A., 2001, «Les homepages, nouvelles écritures de soi, nouvelles lectures de l'autre », Spirale, n²8, Lille, pp. 67-83.

LAHIRE B., 2005, L'esprit sociologique, Paris La découverte.

LECUYER R., 1994, Le développement du concept de soi de l'enfance à la vieillesse, Montréal, Presses de l'Université de Montréal.

LEJEUNE P., 2000, « Les usages du journal intime », Sciences humaines, n¹02, 2000, pp. 30-33.

LENHART A. et FOX S., 2006, "A portrait of the internet new storytellers » Pew internet and American life project, 2006, pp. 1-33,

$<$ http://www.pewinternet.org/PPF/r/186/report_display.asp>, dernière consultation, septembre 2008.

LIN J. et HALAVAIS A., 2006, " Geographical Distribution of Blogs in the United States ", Webology, vol. 3, n4, <http://www.webology.ir/2006/v3n4/a30.html>, dernière consultation, septembre 2008.

LOOSE F., 2000, L'individualisme. Une norme sociale ?, Thèse de doctorat, Clermont-Ferrand II.

MACE E., 2006, La télévision et son double, Paris, Armand Colin.

MARTINOT D., 2001, "Connaissance de soi et estime de soi », Revue des sciences de l'éducation, vol. 30, n³, pp. 483-502. 
MEHL D., 1996, La télévision de l'intimité, Paris, Le Seuil.

MENDRAS H., 1994, La seconde révolution française : 1965-1984, Paris, Gallimard.

MILLER C. et SHEPHERD D., 2004, «Blogging as social action : a genre analysis of the weblog », Blogosphère,

<http://blog.lib.umn.edu/blogosphere/blogging_as_social_action.html>, dernière consultation septembre 2008.

MYERS D. et LAMARCHE L., 1991, Psychologie sociale, Montréal, La Chenelière.

NARDI B., SCHIANO D. et GUMBRECHT M., 2004, «Blogging as Social Activity », dans actes du colloque Proceedings of the Conference on ComputerSupported Cooperative Work, Chicago, The ACM Digital Library, pp. 222-231

NOWSON S. et OBERLANDER J., 2007, « Whose thumb is it anyway ? Classifying author personality from weblog text », dans actes du colloque Proceedings of CoLing/ACL 2006, Sydney, Australie, Association for Computational Linguistics, pp. 1-7, <http://www.ics.mq.edu.au/ snowson/papers/OberNowACL06.pdf>, dernière consultation septembre 2008.

PLEDEL I., 2006, « La blogosphère », Les cahiers du journalisme, $\mathrm{n}^{\circ} 16$, pp. 252-276.

PROST A., 2002, « Comprendre les évolutions », dans BAUDRY, C. SORBETS ET A. VITALIS (dir.), La Vie privée à l'heure des médias, Bordeaux, Presses universitaires de Bordeaux, pp. 23-27.

PROULX S., SENECAL M., 1995, « L'interactivité technique, simulacre d'interaction sociale et de démocratie ? », Technologies de l'information et société, vol. 7-2, pp. 239-255,

<http://revuetis.mshparisnord.org/article.php3?id_article=13, dernière consultation, septembre 2008.

QUERE L., 1989, « La vie sociale est une scène. Goffman revu et corrigé par Garfinkel », dans JOSEPH I (dir.), Le frais parler d'Erving Goffman, Paris, Minuit, pp. 47-82.

ROUQUETTE S., 2001, L'impopulaire télévision populaire, Paris, L'Harmattan.

SEGUY F., 1999, Les produits interactifs et multimédias, Grenoble, Presses Universitaires de Grenoble. 
Les blogs « extimes » : analyse sociologique de l'interactivité des blogs

SENECAL M., 2006, "Interactivité et interaction : sens, usages et pratiques », dans PROULX S., POISSANT L. et SENECAL M. (dir.), Communautés Virtuelles, Laval, Presses de l'Université Laval, pp. 133-146.

SINGLY F., 2000, «Introduction », dans SINGLY F. (dir.), Libres ensemble. L'individualisme dans la vie commune, Paris, Nathan, pp. 5-19.

SINGLY F., 2003, Libres ensemble. L'individualisme dans la vie commune, Paris, Nathan.

TISSERON S., 2001, L'intimité surexposée, Paris, Ramsey.

THIERRY D., 2002, "L'information locale en ligne peut-elle être interactive ? », dans DAMIAN B., RINGOOT R., THIERRY D. et RUELLAN D. (dir.), Inform@tion.local, Paris, l'Harmattan, pp. 247-261.

TREDAN O., 2005, «Les weblogs dans la Cité », Cahier de recherche, n6, pp. 1-10.

WINKIN Y., 2001, Anthropologie de la communication : de la théorie au terrain, Paris, Le Seuil.

ZAJONC R. et HAZEL M., 1982, «Affection and cognitive factors in preference », Journal of Consumer Research, n`9, pp. 123-131.

\section{Pour citer cet article}

Sébastien Rouquette, "Les blogs " extimes " : analyse sociologique de l'interactivité des blogs», Revue tic\&société [En ligne], Tic et santé, Numéros, Varia, mis à jour le : 13/10/2008, URL :

http://revues.mshparisnord.org/lodel/ticsociete/index.php?id=412. 